\title{
The Inflaton and Time in the Matter-Gravity System
}

\author{
A. Tronconi ${ }^{1}$, G.P. Vacca ${ }^{2}$ and G. Venturi ${ }^{3}$ \\ Dipartimento di Fisica - Università di Bologna and INFN - Sezione di Bologna, \\ via Irnerio 46, 40126 Bologna, Italy
}

\begin{abstract}
The emergence of time in the matter-gravity system is addressed within the context of the inflationary paradigm. A quantum minisuperspace-homogeneous minimally coupled inflaton system is studied with suitable initial conditions leading to inflation and the system is approximately solved in the limit for large scale factor. Subsequently normal matter (either non homogeneous inflaton modes or lighter matter) is introduced as a perturbation and it is seen that its presence requires the coarse averaging of a gravitational wave function (which oscillates at trans-Planckian frequencies) having suitable initial conditions. Such a wave function, which is common for all types of normal matter, is associated with a "time density" in the sense that its modulus is related to the amount of time spent in a given interval (or the rate of flow of time). One is then finally led to an effective evolution equation (Schrödinger Schwinger-Tomonaga) for "normal" matter. An analogy with the emergence of a temperature in statistical mechanics is also pointed out.
\end{abstract}

\section{Introduction}

A fundamental property of the interaction of matter and gravity is its spacetime reparametrisation invariance. In particular invariance under time reparametrisation leads to the constraint that the sum of the matter and gravitational Hamiltonians is zero. This implies that time does not appear in the Hamiltonian formulation of the matter-gravity action. However, this does not mean that time does not appear at all in the classical equations of motion, since they involve time derivatives of the dynamical parameters, but rather that there is no absolute time and no clock external to the universe.

\footnotetext{
${ }^{1}$ tronconi@bo.infn.it

${ }^{2}$ vacca@bo.infn.it

3 armitage@bo.infn.it
} 
Such a feature is maintained and becomes even more evident in the canonical quantisation of gravity within the superspace approach [1]. In such an approach the spacetime dynamical variables are the three geometries of spacelike surfaces with their conjugate momenta, while matter is described by the corresponding fields and their conjugate momenta. As a consequence the classical equations correspond to geodesic equations in the manifold of all three geometries [1, 2] (superspace) modified by the presence of a force term. Canonical quantisation then leads to a Schrödingerlike equation (Wheeler-DeWitt, hereafter WDW) without any time derivatives and a corresponding wave function satisfying it.

Clearly, if one considers as a starting point the complete quantum system, it must be possible to re-obtain the usual classical Einstein and quantum matter (SchwingerTomonaga) equations. This last step, however, does not appear to be immediate and has raised considerable interest. In particular the introduction of time has been examined within the Born-Oppenheimer approximation and the semiclassical approximation to gravity both without [3] and with [4, 5] the backreaction of matter. Further it has also been discussed in quite diverse contexts such as through an examination of transition matrix elements of radiative processes occurring in the cosmos [6] or through interaction with the environment [7, 8] or decoherence [9].

The scope of this paper is to examine the above problem by studying the mattergravity quantum system within the context of a mini-superspace model containing a minimally coupled homogeneous scalar field (inflaton) which is known to lead to inflation [10, 11, 12, 13, 14] together with normal matter (which could be the nonhomogeneous modes of the inflaton field or generic inflaton decay products appearing as lighter matter fields).

In the next section we shall study the general minisuperspace-homogeneous inflaton system and obtain an approximate solution, for large scale factor (inflation), suitable for some analytic considerations. Subsequently we shall illustrate the way the presence of normal matter leads to the requirement of an evolution (or time) which is obtained through a coarse graining and relating a gravitational part of the wave function to a measure of temporal density. Lastly, in section four, our results are summarized and discussed.

\section{The gravity-matter system}

As we have anticipated in the introduction we consider the gravity-matter interaction and work with a simple model having an approximate high degree of symmetry. To be definite we shall consider an inflationary cosmology driven by an homogeneous inflaton scalar field, with the presence of extra matter degrees of freedom, which 
will be treated as a small perturbation. Our goal is to study the effective quantum behavior of these latter degrees of freedom in the background of the homogeneous inflaton-gravity system. After having given the classical description of the full system we shall consider the quantized system described by the corresponding WDW equation. The study of the consequences of taking an approximate quantum solution will be of interest for our purposes.

\subsection{The classical system}

We start with a real massive scalar field (inflaton) and other unspecified matter fields minimally coupled to gravity which is described by a Robertson-Walker minisuperspace. The scalar field of mass $m$ is the homogeneous inflaton field $\phi$, typical of chaotic inflation models, while matter fields are generally inhomogeneous, and of typical mass $\mu \leq m$.

On taking $d s^{2}=-d t^{2}+a^{2}(t) d \vec{r}^{2}$, the line element in a flat 3-space, we write the total action with $\hbar=c=1$ :

$$
S=\frac{1}{2} \int d t\left\{-M^{2} a \dot{a}^{2}+a^{3}\left(\dot{\phi}^{2}-m^{2} \phi^{2}\right)+L_{\mu}\right\}
$$

where $M$ is the Plank mass and $L_{\mu}$ is the matter lagrangian, which depends on the scale factor $a$. Let us note that the volume factor has been absorbed by the scaling $a \rightarrow a V^{1 / 3}$. Since $a$ acquires the dimension of a length, the $\vec{r}$ variable is dimensionless just as any momenta $\vec{k}$ arising from a Fourier analysis, further the scalar field $\phi$ has the dimension of a mass.

The classical Hamiltonian is:

$$
H=-\frac{1}{2} \frac{\pi_{a}^{2}}{M^{2} a}+\frac{1}{2 a^{3}}\left(\pi_{\phi}^{2}+m^{2} \phi^{2} a^{6}\right)+H_{\mu} \equiv H_{G}+H_{I}+H_{\mu}
$$

with $\pi_{a}=-M^{2} a \dot{a}, \pi_{\phi}=a^{3} \dot{\phi}$.

\subsection{The quantum system}

The starting point of all our considerations is the quantized matter-gravity system. We shall consider in this section only the homogeneous inflaton degree of freedom minimally coupled to gravity, leaving to section 3 the discussion of the quantum effects on the other matter fields. At the quantum level there is no time in the description of the system since the Hamiltonian, generating the dynamics, is a constraint which annihilates the physical states. The latter can be written as wave 
functions on considering, for example, the $(a, \phi)$ representation. The dynamics is described by a second order partial differential equation which can be interpreted as giving a flow in $a$ of some distribution in $\phi$ given for an initial $a_{0}$.

Physically we shall be interested in configurations with large $a$, typical of a situation which belongs to an advanced stage of the inflationary phase. At the same time we shall have a constraint on the kind of inflaton field quantum distribution which must be compatible with such an inflationary regime.

We therefore start from the quantum (WDW) Einstein equation in the $(a, \phi)$ representation:

$$
\begin{gathered}
\langle a| \otimes\left\langle\phi\left|\left(\hat{H}_{G}+\hat{H}_{I}\right)\right| \Psi\right\rangle \equiv \\
\equiv\left(\frac{1}{2 M^{2}} \frac{\partial^{2}}{\partial a^{2}} \frac{1}{a}-\frac{1}{2 a^{3}} \frac{\partial^{2}}{\partial \phi^{2}}+\frac{m^{2} a^{3}}{2} \phi^{2}\right) \Psi(a, \phi)=0
\end{gathered}
$$

where, for convenience, we have taken a particular ordering for the first term $\left(\frac{\pi_{a}^{2}}{a} \rightarrow\right.$ $\left.\hat{\pi}_{a}^{2} \frac{1}{\hat{a}}\right)$ and $\Psi(a, \phi)$ is the total scale factor-inflaton wave function. Let us note that different orderings, for example manifestly Hermitian ones, will differ by terms of the form $\left(i / a^{2}\right) \hat{\pi}_{a}$ or $\hat{\pi}_{a}\left(i / a^{2}\right)$ which, as we shall later verify, are irrelevant (non-leading) for $a$ large.

It is clear that for a positive definite inflaton Hamiltonian the wave function $\Psi$ will tend to have a strongly oscillatory dependence on $a$. The oscillatory period will be so small that natural scales for the matter dynamics in $a$ will be much longer and a coarse graining, which can be, for example, chosen as an averaging over a period of oscillation in $a$, will be necessary to study the effective matter dynamics. A statistical interpretation as given in studies of the probability distributions in the hydrogen atom [15] gives further insight. We shall develop this idea in section 3 and concentrate in the following on studying the oscillatory behavior of the "universe" wave function.

It is convenient to write an expansion on a complete basis of the Hilbert space of the states of the inflaton field associated with the Hamiltonian $\hat{H}_{I}$ :

$$
\Psi(a, \phi)=a \sum_{n} \psi_{n}(a) u_{n}(a, \phi) \equiv a \psi(a) \sum_{n} c_{n}(a) u_{n}(a, \phi) \equiv a \psi(a) u(a, \phi)
$$

on using the eigenstates of the inflaton Hamiltonian $\hat{H}_{I}$ one has:

$$
\hat{H}_{I}\left|u_{n}\right\rangle=m\left(n+\frac{1}{2}\right)\left|u_{n}\right\rangle .
$$


Substitution of (41) into (3) leads to (henceforth $\partial_{a} \equiv \frac{\partial}{\partial a}$ ):

$$
\begin{gathered}
u(a, \phi) \partial_{a}^{2} \psi(a)+2 \partial_{a} u(a, \phi) \partial_{a} \psi(a)+\psi(a) \partial_{a}^{2} u(a, \phi)+ \\
+2 M^{2} \psi(a)\left(-\frac{1}{2 a^{2}} \frac{\partial^{2}}{\partial \phi^{2}}+\frac{m^{2} a^{4}}{2} \phi^{2}\right) u(a, \phi)=0
\end{gathered}
$$

Let us comment on the expansion in (4), and its statistical interpretation in quantum mechanics. We note that $\left|c_{n}\right|^{2}$ is the probability that a state of $n$ quanta is created (observed) and the $c_{n}$ arose through the extraction of a common factor $\psi(a)$ from the $\psi_{n}(a)$. That is the superposition of the different $n$ quantum number configurations occurred after extracting the common factor from the sum of the products of each $n$ quantum state with its corresponding universe wave function $\psi_{n}(a)$. Further we expect the homogeneous inflatonic matter initial state to be such as to lead to inflation. Thus we assume that the initial conditions are associated with the creation (considered as the inverse of a decay process) of a large average number $\bar{n}$ of quanta in an initial interval of $a$. This can then be imagined as occurring through a Poisson process so that the initial state associated with the interval of $a$ is related to a Poisson distribution.

Thus, instead of having a sequence of random creations of $n$ quanta (with average $\bar{n})$ corresponding to a sequence of inflationary processes in successive infinitesimal intervals of $a$, we consider a statistical average, that is a superposition of the different possible quanta numbers which can occur in the interval of $a$ leading to inflation. Subsequently $a$ will undergo a rapid change due to inflation.

This distribution is familiar in quantum mechanical oscillators. It is associated with coherent states which, for large average occupation numbers, describe an almost classical behavior. We shall discuss the above assumption in the next section and find that for large $a$ it is a reasonable one, independently of any possible interpretation of its origin.

\subsection{Approximating the quantum inflaton-gravity system}

This section is devoted to the construction of an approximate solution of the WDW which corresponds to an inflationary situation. As a next step, in the following section, we shall make use of the results of this section in order to obtain some analytical insights on the dynamics of other matter degrees of freedom and show how a concept of time arises naturally, a phenomenon which in any case appears to be independent of the approximation employed.

To extract an approximate solution two procedures are possible: either try to solve directly the partial differential equation (6) or make an ansatz for $u(a, \phi)$ and 
show that it leads to an approximate solvable differential equation for $\psi(a)$. We shall choose the latter approach.

For convenience we rewrite the inflaton Hamiltonian $\hat{H}_{I}(a) \equiv\left\langle a\left|\hat{H}_{I}\right| a\right\rangle$ in terms of inflaton creation and annihilation operators:

$$
\hat{H}_{I}(a) \equiv \frac{\hat{\pi}_{\phi}^{2}}{2 a^{3}}+\frac{m^{2} a^{3}}{2} \hat{\phi}^{2}=m\left(b^{\dagger} b+\frac{1}{2}\right),
$$

where

$$
b=\sqrt{\frac{m a^{3}}{2}}\left(\hat{\phi}+\frac{i}{m a^{3}} \hat{\pi}_{\phi}\right), \quad b^{\dagger}=\sqrt{\frac{m a^{3}}{2}}\left(\hat{\phi}-\frac{i}{m a^{3}} \hat{\pi}_{\phi}\right)
$$

and the ansatz for $u(a, \phi)$ will be:

$$
u(a, \phi)=\langle\phi \mid \alpha(a)\rangle,
$$

where $|\alpha(a)\rangle$ is a coherent state of the inflaton, defined by

$$
b \mid \alpha(a))\rangle=\alpha(a)|\alpha(a)\rangle
$$

with $\alpha(a)=\sqrt{\frac{m \phi_{0}^{2} a^{3}}{2}}$. From eqs. (9) and (10) $u(a, \phi)$ must satisfy the differential equation:

$$
\left(\frac{1}{m a^{3}} \frac{\partial}{\partial \phi}+\phi-\phi_{0}\right) u(a, \phi)=0
$$

where $\phi_{0}$ is a free parameter. Equation (11) has the familiar normalized solution:

$$
u(a, \phi)=\left(\frac{m a^{3}}{\pi}\right)^{\frac{1}{4}} \exp \left[-\frac{m a^{3}}{2}\left(\phi-\phi_{0}\right)^{2}\right]
$$

which is a simple gaussian peaked around $\phi_{0}$ with a width which decreases as the scale factor $a$ increases. The values of the parameter $\phi_{0}$ are constrained in order to lead to an inflationary regime [13] $\left(\bar{n}=\alpha(a)^{2}\right)$, since such a quantum coherent state description can be related to the classical analysis of the inflaton system.

Let us now study the consequences of such a choice for $u$. If we substitute the expression (12) into equation (6) and calculate the contributions of the different derivatives we obtain:

$$
\begin{aligned}
& \partial_{a} u(a, \phi)=\left[\frac{3}{4 a}-\frac{3 m a^{2}}{2}\left(\phi-\phi_{0}\right)^{2}\right] u(a, \phi) \\
& \partial_{a}^{2} u(a, \phi)=\left[-\frac{3}{16 a^{2}}-\frac{21 m a}{4}\left(\phi-\phi_{0}\right)^{2}+\frac{9 m^{2} a^{4}}{4}\left(\phi-\phi_{0}\right)^{4}\right] u(a, \phi), \\
& \frac{\partial^{2}}{\partial \phi^{2}} u(a, \phi)=\left[-m a^{3}+m^{2} a^{6}\left(\phi-\phi_{0}\right)^{2}\right] u(a, \phi) .
\end{aligned}
$$


A key point is that we are interested in solutions of (3), describing an advanced stage of inflation $(a \gg 1)$, since in this phase we wish to study how normal matter behaves. In this limit all the terms in the expressions (13) 15) which are of the form $\left(\phi-\phi_{0}\right)^{n} u(a, \phi)$ with $n>0$, are non-leading by powers of $a$ with respect to $u(a, \phi)$ since

$$
\max \left[\left|\phi-\phi_{0}\right|^{n} u(a, \phi)\right] \sim\left(\frac{n}{m a^{3}}\right)^{\frac{n}{2}} \exp \left[-\frac{n}{2}\right] \max [u(a, \phi)] ;
$$

in other words one has:

$$
\begin{aligned}
& \partial_{a} u(a, \phi)=O\left(a^{-1}\right) u(a, \phi), \\
& \partial_{a}^{2} u(a, \phi)=O\left(a^{-2}\right) u(a, \phi), \\
& \frac{\partial^{2}}{\partial \phi^{2}} u(a, \phi)=O\left(a^{3}\right) u(a, \phi) .
\end{aligned}
$$

On just retaining the leading contributions in (6) one has:

$$
\left[\partial_{a}^{2} \psi(a)+m^{2} M^{2} \phi^{2} a^{4} \psi(a)\right] u(a, \phi)=0
$$

where $u(a, \phi)$ has support in a tiny region around $\phi_{0}$, due to the large values of $a$. We have also used the fact that any term of $O(1 / a) \psi(a)$ is negligible with respect to $\partial_{a} \psi(a)$ for large $a$, as can be verified on using the solution found for $\psi(a)$. Further additional terms such as $\partial_{a}\left(1 / a^{2}\right)$ or $\left(1 / a^{2}\right) \partial_{a}$, due to different orderings in the gravitational kinetic term, are non-leading when acting on $a u(a, \phi)$ (see eq. (17) and (18) ) and are also negligible with respect to $\partial_{a}^{2} \psi$ when acting on the solution found for $\psi(a)$. Therefore the choice of ordering is irrelevant for large $a$. Finally one may rewrite (20) as:

$$
\partial_{a}^{2} \psi(a)+m^{2} M^{2} \phi_{0}^{2} a^{4} \psi(a)=0
$$

or on changing to the variable $y=a^{2}$ (again apart from a non-leading term for $a$ large),

$$
\frac{\partial^{2}}{\partial y^{2}} \psi(y)+\frac{m^{2} M^{2} \phi_{0}^{2}}{4} y \psi(y)=0
$$

A general solution, in terms of the Airy functions $A_{i}$ and $B_{i}$, for eq. (22) is:

$$
\psi(y)=C_{1} A i(y)+C_{2} B i(y) \rightarrow y^{-\frac{1}{4}}\left[D_{1} \sin \frac{m M \phi_{0}}{3} y^{\frac{3}{2}}+D_{2} \cos \frac{m M \phi_{0}}{3} y^{\frac{3}{2}}\right]
$$

as $y \rightarrow \infty$, or:

$$
\psi(a) \sim a^{-\frac{1}{2}}\left[D_{1} \sin \frac{m M \phi_{0}}{3} a^{3}+D_{2} \cos \frac{m M \phi_{0}}{3} a^{3}\right]
$$


for $a \gg 1$ where $D_{1}$ and $D_{2}$ are complex numbers. The oscillatory behavior is encoded in $\psi$, even if at an approximate level (the solution is not exact). Let us note that the $D_{i}\left(C_{i}\right)$ in (23) can be determined by the initial conditions. For example, $C_{1}=-i$ and $C_{2}=1$ corresponds to the Vilenkin initial wave function of the universe, while if $C_{1}=1$ and $C_{2}=0$ one has the Hartle-Hawking choice.

One may ask what would be the effect of a perturbation such as the addition of a small contribution of $n$ inflaton quanta. Because of the rapid oscillatory behavior of the gravitational wave function and the fast increase of $\bar{n}$ as $a \rightarrow \infty$ such a contribution will be quickly washed out. Thus our "coherent" state will remain dominant during inflation. Nonetheless some correction are certainly expected. For example in the classical limit (one has the classical Einstein equation) there are nonleading non power-like corrections to the oscillatory phase which are expcted to be of a logarithmic nature. We shall comment more on this in the following section.

\section{The appearance of time in the description of matter}

Let us at this point discuss the dynamics of "normal" matter fields. The quanta of these fields, associated with matter particles, are assumed to contribute as a small perturbation in the total Hamiltonian where the inflaton (homogeneous mode condensate) and gravity are dominant and whose classical expression was given in (2). In order to study this quantum problem one has to enlarge the corresponding Hilbert space of the physical states, in which the inflaton-gravity quantum state is only negligibly affected by the presence of "normal" matter, and consider the following extended quantum equation for the factorized wave function:

$$
\begin{gathered}
{\left[\frac{1}{2 M^{2}} \frac{\partial^{2}}{\partial a^{2}} \frac{1}{a}-\frac{1}{2 a^{3}} \frac{\partial^{2}}{\partial \phi^{2}}+\frac{m^{2} a^{3}}{2} \phi^{2}+\hat{H}_{\mu}\right] \Psi \chi \equiv} \\
\equiv\langle a| \otimes\left\langle\phi\left|\otimes\left\langle\varphi\left|\left(\hat{H}_{G}+\hat{H}_{I}+\hat{H}_{\mu}\right)\right| \Psi\right\rangle \otimes\right| \chi\right\rangle=0,
\end{gathered}
$$

where we have collectively denoted with $\varphi$ all the matter fields (bosonic and fermionic) and $\chi(\varphi, a)$ describes their quantum state in some representation.

The first term in eq. (25) $\hat{H}_{G}$ acts on both $\Psi(a, \phi)$ and $\chi(\varphi, a)$, the last $\left(\hat{H}_{\mu}\right)$ only on $\chi$ and $\hat{H}_{I}$ only on $\Psi$. On using eq. (3) for $\Psi$ we can obtain an equation for the "normal" matter wave function $\chi$ :

$$
2 \partial_{a}\left(\frac{\Psi}{a}\right) \partial_{a} \chi+\frac{\Psi}{a} \partial_{a}^{2} \chi+2 M^{2} \Psi \hat{H}_{\mu}(\varphi, a) \chi=0
$$


where, for sake of compactness, $\hat{H}_{\mu}(\varphi, a) \equiv\left\langle a\left|\otimes\left\langle\varphi\left|\hat{H}_{\mu}\right| a\right\rangle \otimes\right| \varphi\right\rangle$. Further on defining $\tilde{\psi}(a, \phi) \equiv \psi(a) u(a, \phi)$ and bearing in mind (4) one has:

$$
2 \frac{\partial_{a} \tilde{\psi}}{\tilde{\psi}} \partial_{a} \chi+2 a M^{2} \hat{H}_{\mu}(\varphi, a) \chi+\partial_{a}^{2} \chi=0
$$

Let us now examine the different terms in (27) while assuming the presence of a finite number $N$ of quanta of normal matter. It is convenient to consider a free matter (bosonic or fermionic) field. Then it is clear that the eigenvalues of the Hamiltonian will be independent of $a$ as $a \rightarrow \infty$. For example a state on $N$ bosonic quanta of momentum $k$ leads to a contribution $\sim \omega_{k}\left(N+\frac{1}{2}\right)$ where $\omega_{k}^{2}=\frac{k^{2}}{a^{2}}+\mu^{2}$ (apart from a normal ordering subtraction). The last term is then proportional to:

$$
\left(\sum_{L}\left\langle N\left|\overleftarrow{\partial}_{a}^{2}\right| L\right\rangle\left\langle L\left|\vec{\partial}_{a}^{2}\right| N\right\rangle\right)^{\frac{1}{2}} \propto \frac{N^{2}}{a^{2}}
$$

and is negligible with respect to the second for large $a$.

The first term in (27) contains all the information related to the behavior of the gravity-inflaton wave function which is highly oscillatory in $a$ with a typical period much less than a Planck time (length) (see below). Since any physical phenomena of interest for matter are related to scales above (or at least not below) the Planck time $M^{-1}$, it is very natural to obtain an effective equation by means of a course graining. In particular we shall implement it by averaging over an oscillation period in $a$.

\subsection{Approximate analysis}

In order to compare the first two terms of (27) we consider the explicit expression of the approximate solution for $\Psi=a \tilde{\psi}$ derived in the preceding section and write:

$$
\frac{\partial_{a} \tilde{\psi}}{\tilde{\psi}} \equiv \frac{\partial_{a} \psi(a)}{\psi(a)}+\frac{\partial_{a} u(a, \phi)}{u(a, \phi)}
$$

On recalling eq. (13) the second term behaves as $\frac{\partial_{a} u(a, \phi)}{u(a, \phi)} \sim \frac{1}{a}$ while the first term is asymptotically given by

$$
\frac{\partial_{a} \psi(a)}{\psi(a)}=\frac{\partial y}{\partial a} \frac{\partial_{y} \psi(y)}{\psi(y)} \sim \frac{m M \phi_{0} a^{\frac{3}{2}}}{\psi(a)}\left[D_{1} \cos \frac{m M \phi_{0}}{3} a^{3}-D_{2} \sin \frac{m M \phi_{0}}{3} a^{3}\right] .
$$


The crucial point is now to estimate the ratio (30): $\psi(a)$ and its derivative are products of a highly oscillating function of $a$ with some power of $a$. Let us consider the $a$-dependent period of oscillation $\Delta a$

$$
\Delta a \sim \frac{2 \pi}{m M \phi_{0} a^{2}}
$$

Since $M$ is the Plank mass, $m \sim 10^{-6} M \ll M, \phi_{0} \sim M$ and $a \gg M^{-1}$ (by many orders of magnitude) during the inflationary phase for $a$ large, one obtains the inequality

$$
\Delta a \ll M^{-1} .
$$

Thus one may estimate (30) by averaging over an interval $[a, a+\Delta a]$ which is much shorter than a Planck length:

$$
I_{a v}=\frac{1}{\Delta a} \int_{a}^{a+\Delta a} d \alpha\left(m M \phi_{0} \alpha^{2}\right) \frac{\left[D_{1} \cos \frac{m M \phi_{0}}{3} \alpha^{3}-D_{2} \sin \frac{m M \phi_{0}}{3} \alpha^{3}\right]}{\left[D_{1} \sin \frac{m M \phi_{0}}{3} \alpha^{3}+D_{2} \cos \frac{m M \phi_{0}}{3} \alpha^{3}\right]} .
$$

The coarse-graining integral (333) is well defined for any complex $D_{1}$ and $D_{2}$, except for a zero measure case where their ratio is real, since the denominator in the integrand may vanish. To obtain a finite result one may then consider the principal value of the integral. We shall see that, except for the latter case and depending on the initial conditions discussed at the end of section 2, one is able to introduce a time evolution. In particular one finds that Vilenkin type wave functions allow one to introduce a time, while Hartle-Hawking ones do not. On defining a new integration variable $x=\frac{m M \phi_{0}}{3} \alpha^{3}$ one may write:

$$
I_{a v}=\frac{m M \phi_{0}}{2 \pi} a^{2} \int_{0}^{2 \pi} d x \frac{\left[D_{1} \cos x-D_{2} \sin x\right]}{\left[D_{1} \sin x+D_{2} \cos x\right]},
$$

which can be evaluated in the complex plane by using Jordan's Lemma and the Residue Theorem. In fact on setting $z=\exp i x$ one has $\cos x=\frac{1}{2}\left(z+\frac{1}{z}\right), \sin x=$ $\frac{1}{2 i}\left(z-\frac{1}{z}\right), d x=-\frac{i}{z} d z$ and the integral (34) becomes:

$$
I_{a v}=\frac{m M \phi_{0}}{2 \pi i} a^{2} \oint \frac{d z}{z} \frac{i D_{1} z^{2}+i D_{1}-D_{2} z^{2}+D_{2}}{D_{1} z^{2}-D_{1}+i D_{2} z^{2}+i D_{2}}
$$

The function in the integrand (let us denote it by $F(z)$ ) has three singularities, at $z_{0}=0$ and $z_{1,2}= \pm \sqrt{\frac{D_{1}-i D_{2}}{D_{1}+i D_{2}}} \equiv \pm A e^{i \theta}: z_{0}$ will always lie within the path of integration (the circumference of radius 1 centred on the origin of the complex plane) 
while $z_{1,2}$ generally will not. Only if $D_{1}$ and $D_{2}$ have a real ratio one has $A=1$ and $z_{1,2}$ are on the circumference. If one evaluates the residues of $F(z)$ at $z_{0,1,2}$ one obtains:

$$
\left.\operatorname{ResF}(z)\right|_{z=z_{0}}=-i,\left.\quad \operatorname{ResF}(z)\right|_{z=z_{1,2}}=i
$$

To summarize, one can distinguish between 3 cases:

when $0<A<1$ :

$$
I_{a v}=m M \phi_{0} a^{2}\left(\left.\operatorname{ResF}(z)\right|_{z=z_{0}}+\left.\operatorname{ResF}(z)\right|_{z=z_{1}}+\left.\operatorname{ResF}(z)\right|_{z=z_{2}}\right)=i m M \phi_{0} a^{2}
$$

when $A>1$ :

$$
I=m M \phi_{0} a^{2}\left(\left.\operatorname{Res} F(z)\right|_{z=z_{0}}\right)=-i m M \phi_{0} a^{2}
$$

when $A=1$ the calculations are slightly different since one has to deform the path of integration to avoid the two singularities and then let the deformation tend to zero. For instance, the path could turn around $z_{1,2}$ following: $z_{1,2}(\gamma)=z_{1,2}+\epsilon e^{i \gamma}$ with $\gamma$ going from $\theta-\frac{\pi}{2}$ to $\theta-\frac{3 \pi}{2}$ around $z_{1}$ and from $\theta+\frac{\pi}{2}$ to $\theta-\frac{\pi}{2}$ around $z_{2}$ :

$$
\begin{aligned}
I & =m M \phi_{0} a^{2}\left(\left.\operatorname{Res} F(z)\right|_{z=z_{0}}\right)-\lim _{\epsilon \rightarrow 0}\left[\frac{m M \phi_{0}}{2 \pi i} a^{2}\left(\int_{z_{1}(\gamma)} d z F(z)+\int_{z_{2}(\gamma)} d z F(z)\right)\right]= \\
& =-i m M \phi_{0} a^{2}-\left[\frac{m M \phi_{0}}{2 \pi i} a^{2}(\pi+\pi)\right]=0
\end{aligned}
$$

Thus,unless $A=1$, coarse-graining of the ratio (30) leads to a non zero result, in which case the dominant contribution in (29) comes from the first term and one has:

$$
\frac{\partial_{a} \tilde{\psi}}{\tilde{\psi}} \sim \pm i m M \phi_{0} a^{2}
$$

We must further estimate $\partial_{a} \chi$, again for $\mathrm{N}$ quanta, obtaining for its magnitude:

$$
\left[\left\langle N\left|\overleftarrow{\partial}_{a} \vec{\partial}_{a}\right| N\right\rangle\right]^{\frac{1}{2}} \equiv\left[\sum_{L}\left\langle N\left|\overleftarrow{\partial}_{a}\right| L\right\rangle\left\langle L\left|\vec{\partial}_{a}\right| N\right\rangle\right]^{1 / 2} \propto \frac{N}{a}
$$

As for the last term, we have shown it to be highly suppressed.

On just retaining the two leading terms in eq.(27) one obtains

$$
\pm i \frac{m \phi_{0}}{M} a \partial_{a} \chi=\hat{H}_{\mu}(\varphi, a) \chi
$$


Therefore we are left with a parabolic PDE which describes the evolution of "normal" matter with respected to $a$. On just defining a time $t$ according to $\pm i m \phi_{0} / M \partial_{\text {loga }} \chi \equiv$ $i \partial_{t} \chi$ one obtains a Schrödinger equation describing the quantum mechanical evolution of matter, or a Schwinger-Tomonaga equation

$$
i \partial_{t} \chi=\hat{H}_{\mu}(\varphi, a) \chi .
$$

Let us note that this is equivalent to interpreting the coefficient of $i \partial_{a} \chi$ on the l.h.s. of (42) as $\dot{a}$, the rate of expansion seen by the "normal" matter (observers + clocks). In particular the approximate analysis employed here shows a DeSitter type expansion. In general an observer made of "normal" matter, using clocks made of normal matter, can only measure any change of state with respect to the evolution of the $t$ introduced above as the parameter of the Schrödinger equation (421). In other words it is the presence of normal matter which leads naturally to the introduction of a time. The sign in eq. (42) depends on the initial conditions which characterize the solution $\psi(a)$. With generic conditions, one of the two cases is realized and this, in our approach, is related to the definition of the flow of time.

\subsection{Beyond the approximation}

The result we have obtained is reasonable, especially considering the approximations made during the inflationary phase. A different scale dependent evolution seen by matter, such as the one obtained in the classical limit of the chaotic inflation model, could be easily recovered by a very mild correction (logarithmic) to the coefficient of $i \partial_{a} \chi$, which allowes for the difference between a constant and an approximate linearly decreasing (in time) Hubble parameter.

For the general case, with a strongly peaked inflaton wave function, independently of any particular approximation, the oscillatory wave function in the large $a$ region can be written as $\tilde{\psi}(a)=\rho(a) \exp i \beta(a)$, where $\rho$ is an oscillating real function. For most initial conditions, as in the approximate solution, such a function never vanishes except possibly at an extreme of the oscillation. Only a zero measure set of initial conditions will not satisfy this property leading to the same consequences as already discussed in the previous subsection. After the course-graining integration one can therefore make the correspondence:

$$
\frac{1}{a M^{2}} \frac{\partial_{a} \tilde{\psi}}{\tilde{\psi}} \partial_{a}=\frac{1}{a M^{2}}\left(\frac{\rho^{\prime}(a)}{\rho(a)}+i \beta^{\prime}(a)\right) \partial_{a} \approx \frac{i}{a M^{2}} \beta^{\prime}(a) \partial_{a} \rightarrow i \partial_{t}
$$

where a prime denotes differentiation with respect to $a$, since after course-graining the term $\rho^{\prime}(a) /(a \rho(a)) \partial_{a}$ is always negligible and the gradient of the phase is unaffected. 
From this formula one can recover the previous approximate result. Indeed, for example, for the case of the Vilenkin type wave function one has $\beta(a)=m M \phi_{0} a^{3} / 3$. Again time is defined in terms of the inverse of the gradient of the phase of the $\psi$ wave function with respect to the scale factor, and in particular $d t \equiv\left(a M^{2} / \beta^{\prime}(a)\right) d a$.

Until now we have worked with the amplitude and not the probability density, however for large $a$ a probability density analysis leads to the same result. One may easily convince oneself of this on examining the probability current, which satisfies the relation

$$
J_{a} \propto \tilde{\psi}^{*} \partial_{a} \tilde{\psi}-\tilde{\psi} \partial_{a} \tilde{\psi}^{*} \approx 2 i \rho^{2} \partial_{a} \beta \propto \partial_{a} \tilde{\psi}
$$

since, in the limit we consider and on repeating the previous steps, the terms leading to differences are negligible. This means that the density flux is proportional to the gradient of the phase and the fundamental condition for time to arise, in our framework, is then a non negligible coarse-grained probability current.

The presence of an effective time evolution for matter arises from a mechanism similar to one already observed in the analysis of the classical limit of quantum systems, such as the hydrogen atom [15], in the sense that the quantum probability as a function of $a$ is similar to the measure of temporal density in a classical orbit. This fact has been studied for the stationary quantum eigenstates of the hydrogen atom (with two particular fixed values of the angular momenta and large principal quantum number $n$ ) one of which presents a radial highly oscillatory behavior. On course graining (in particular on applying the Riemann-Lebesgue Lemma) one is able to recover the classical trajectory related to the given angular momenta. Indeed the classical trajectory is related to a classical spatial probability distribution of a particle in terms of the inverse of its speed (the fraction of time spent in a spatial interval is a measure of the probability density). There is a deep connection between the above example and the situation present in the matter-gravity system.

As a side remark we note that for the particular case wherein one performs a WKB analysis in order to obtain the classical limit of equation (3) (since this leads to the Hamilton-Jacobi equation), $\tilde{\psi}$ is characterized by $\rho \sim\left(\beta^{\prime}\right)^{-1 / 2}$ (with $\beta$ the classical action) which does not oscillate and the usual relation $\dot{a} \sim \pi_{a} / a \sim \beta^{\prime}(a) / a$ is found. In any case we stress that the relation in (44) is quite general, a much wider class of states for the inflaton-minisuperspace system can lead to an effective time evolution of "normal" matter.

It is worth noting that we always obtain in the course-grained differential equation for "normal" matter, which constitutes a perturbation in the whole Hamiltonian system, a factorized term of the form $f(a) \partial_{a} \chi$. In such a term $f(a)$ is related to the variation of the universe wave function (minisuperspace+inflaton) regarded as a system separate from "normal" matter. It therefore leads to a universal time for all 
the "normal" matter since it is a common factor for all types of "normal" matter. One has an analogy with the introduction of a temperature in statistical mechanics if one thinks of the minisuperspace-inflaton system in dynamical equilibrium as a sort of "heat" bath and normal matter (a perturbation) in "thermal" equilibrium with the "bath" (reservoir), in the sense that is acquires from the "bath" a time (in analogy with temperature in statistical mechanics).

A short comment also on a possible highly quantum mechanical situation wherein the initial condition leads to a coherent superposition of states described by a wave function of the inflaton $\phi$ consisting of well separated, almost gaussian, peaks. A similar definition of time can be extracted on repeating the analysis performed above using a probability density and its flux, constructing a density matrix and subsequently tracing over the possible inflaton states. Indeed the different superimposed states are almost orthogonal and one is finally left with an average gradient of the phase which plays an analogous role to the single peak case in the construction of an effective Schrödinger equation for matter. The application of course-graining techniques in the context of quantum cosmology has also been considered by other authors [8, 9], for example in the study of the wave function of a minisuperspace model by using the Wigner function approach, where diverse kind of coarse-graining are discussed in the search for the emergence of a classical behavior for gravity.

In our quantum mechanical framework it is tempting to say that time "exists" only as far as "normal" matter or inhomogeneous modes for the inflaton or metric fluctuations are concerned. The homogeneous minisuperspace gravity and inflaton condensate system may still be in a quantum state, which is practically unobservable in the sense specified above for the other physical degrees of freedom ("normal" matter and inhomogeneous modes of inflaton and gravity). Indeed any observer, made of "normal" matter will only see a classical time dependent scale factor of the universe. Lastly let us emphasize that the presence of inflatonic matter driving the universe is crucial: in a chaotic inflation model for which $\phi_{0}=0$ [13] one would not obtain suitable conditions for time to evolve.

\section{Conclusions}

As we mentioned in the introduction the problem of the emergence of time in the context of the matter-gravity system has already drawn considerable attention. Nonetheless we feel that the issue is worth revisiting in a cosmological minisuperspace context, in particular within the inflationary paradigm [10, 11, 12, 14. Inflation gives a framework in which to pose basic cosmological questions.

Associated with inflation is the early universe dominance of a vacuum energy 
density effectively described by a hypothetical scalar field called inflaton. In particular we have considered a simple model of chaotic inflation consisting of a minimally coupled massive scalar field in a flat 3-space with inflation being driven by the homogeneous mode. We then start from the quantum minisuperspace-homogeneous scalar field system. Such a quantum system has been previously studied within a BornOppenheimer approach and shown to lead to inflation even for non classical initial states [13. Our present approach and goal are quite different: we study this system, in an inflationary regime, in order to understand its influence on the dynamics of other degrees of freedom which we call "normal" matter.

The quantum minisuperspace homogeneous-inflaton system cannot be solved exactly, however some of its properties can be obtained through the use of a suitable ansatz for the initial state which allowes us to find an approximate solution for $a$ large. The results we obtain are not a consequence of our approximation, which certainly aids us in suggesting and understanding the basic mechanisms, but reflect a general structure for the effective dynamics of "normal" matter.

Our approximate approach consists in expanding the total matter-gravity wave function on a basis of the states associated with different numbers of homogeneous inflaton quanta. Subsequently, a common minisuperspace wave function is extracted from the expansion and the modulus squared of the coefficients multiplying the states with different number of inflaton quanta is then interpreted as the probability for the creation of such a state in the initial interval leading to inflation, bearing in mind, of course, that such a number is large in order for suitable inflation to take place.

The search for a suitable ansatz for the initial state may be related to a creation process for a large number of quanta. Such a mechanism can be considered as analogous to the inverse of a decay process, that is a Poisson process, without concerning oneself as what it is (the equivalent of the decay products)that leads to the creation. The initial conditions are then associated with a Poisson distribution of states with diverse numbers $n$ of inflaton quanta and with average number of quanta $\bar{n}$. This can be seen either as an initial statistical distribution in which each $n$ quantum creation is associated with an inflationary gravitational wave function or a sequence of inflationary processes in successive intervals of $a$ which are however so close that $a$ is approximately constant. In all cases a common part of the gravitational wave function is extracted leaving the different quanta numbers states with their Poisson weights which then combine into a coherent state.

The quantity which is then studied is the common gravitational wave function. It is found to satisfy the same Airy equation for $a$ large, independently of any ordering chosen for the gravitational kinetic term, leading to a strong oscillatory behavior having a frequency many orders of magnitude greater than the Planck one. The 
oscillatory behavior of the complete wave function is the main feature we use and we stress that is not related to the approximation made but just to initial conditions such as those leading to inflation.

One may at this point introduce normal matter which should be regarded as a small perturbation with respect to the contribution of the homogeneous inflaton mode. Again in this context $a$ is considered large and an effective "flow", due to the nature of the gravity-inflaton wave function, can be associated to the existence of time for normal matter. In order to obtain this, a "coarse graining" was performed so as to smooth out the effects of the gravitational wave function oscillations at ultra-Planckian frequencies (for the atomic case see [15]), to which normal matter is insensitive. It is also worth noting that the common gravitational wave function term leads to the introduction of a "time density" (the magnitude of the gravitational wave function in an interval of $a$ being related to the "time" spent in that interval) which is universal, i.e. independent of the type of normal matter associated with it. The paradigm of time developed here arises from a novel point of view, but nonetheless leads to the usual time that one considers in the WKB limit of the gravity-inflaton system.

Moreover we observe that a new interesting possibility is the emergence of a time even with gravity and the inflaton in quantum regimes: it is a consequence of the fact that normal matter cannot see quantum fluctuations below the Planck size but just experiences an evolution with respect to a function of the scale factor, associated with the speed of inflation. In such a framework time only exists for normal matter which evolves according to its position on the gravitational wave function.

In a sense, and particularly on considering the approximate analysis, this emergence of a universal time reminds us of the derivation of a Boltzmann distribution and a temperature for systems obtained by placing them in contact with a large heat bath where it is the density of energy levels of the heat bath that is related to the (common) temperature of systems in contact with it, different energy level densities for the reservoir being associated with different temperatures. Analogously, for us, normal matter is in contact with a gravitational wave function generated by the homogeneous mode inflaton background (reservoir- "time bath") and it is that wave function with suitable initial conditions that generates a "time density" and "flow" which leads to the usual evolution of matter corresponding to the value of the matter wave function for different values of $a$ in the gravitational wave function.

Lastly let us note that we have studied the introduction of time during the inflationary era with gravitation being driven by the homogeneous inflaton Hamiltonian. Clearly one may ask what happens at the end of inflation when presumably all (or most or some) of the homogeneous modes have decayed into lighter matter. In such 
a case gravitation will just be driven by the mean energy of all matter (including a residual cosmological constant) and the resulting gravitational wave function will introduce a common time for any small sub-system which may be regarded as a perturbation of the whole.

\section{Acknowledgement}

We wish to thank R. Brout for several clarifying discussions.

\section{References}

[1] Wheeler J. A., Relativity, Groups and Topology (Les Houches Lectures, 1963), Ed. C. DeWitt and B. DeWitt (New York, Gordon and Breach) (1964).

[2] B. S. DeWitt,Phys. Rev. 160, 113 (1967).

[3] T. Banks, Nucl. Phys. B 249, 332 (1985).

[4] R. Brout,Found. Phys. 17, 603 (1987); Z. Phys. 68, 339 (1987);

R. Brout, G. Horwitz and D. Weil, Phys. Lett. B 192, 318 (1987).

[5] R. Brout and G. Venturi, Phys. Rev. D 39, 2436 (1989).

[6] R. Brout and R. Parentani, Int. J. of Mod. Phys. D 8, 1 (1999).

[7] J. S. Briggs and J. M. Rost, quant-ph/9902035.

[8] E. Calzetta, M. Castagnino and R. Scoccimarro, Phys. Rev.D 45, 2806 (1992).

[9] S. Habib and R. Laflamme, Phys. Rev. D 42, 4056 (1990).

[10] R. Brout, F. Englert and E. Gunzig, Gen. Rel. Grav. 10,1 (1979); A.A. Starobinsky, Phys. Lett. B 91, 99 (1980).

[11] A. H. Guth, Phys. Rev.D 23, 347 (1981).

[12] A. D. Linde,Phys. Lett.B 129, 177 (1983).

[13] F. Finelli, G. P. Vacca and G. Venturi, Phys. Rev. D 58, 103514 (1998).

[14] J. Garcia-Bellido, arXiv:hep-ph/0210050.

[15] E. G. Peter Rowe, European J. Physics 8, 81 (1987). 